\title{
A microsporidian parasite of the genus Spraguea in the nervous tissues of the Japanese anglerfish Lophius litulon
}

\author{
Mark A. Freeman, Hiroshi Yokoyama and Kazuo Ogawa
}

Department of Aquatic Bioscience, Graduate School of Agricultural and Life Sciences, The University of Tokyo, Yayoi 1-1-1, Bunkyo, Tokyo 113-8657, Japan

Key words: Spraguea, microsporidia, Lophius, nervous tissue

\begin{abstract}
In the present study, a high percentage of Japanese anglerfish, Lophius litulon (Jordan, 1902), contained a microsporidian infection of the nervous tissues. Xenomas were removed and prepared for standard wax histology and transmission electron microscopy (TEM). DNA extractions were performed on parasite spores and used in PCR and sequencing reactions. Fresh spores measured $3.4 \times 1.8 \mu \mathrm{m}$ and were uniform in size with no dimorphism observed. TEM confirmed that only a single developmental cycle and a single spore form were present. Small subunit (SSU) rDNA sequences were $>99.5 \%$ similar to those of Spraguea lophii (Doflein, 1898) and Glugea americanus (Takvorian et Cali, 1986) from the European and American Lophius spp. respectively. The microsporidian from the nervous tissue of L. litulon undoubtedly belongs in the genus Spraguea Sprague et Vávra, 1976 and the authors suggest a revision to the generic description of Spraguea to include monomorphic forms and the transfer of Glugea americanus to Spraguea americana comb. n. Since no major differences in ultrastructure or SSU rDNA sequence data exist between Spraguea americana and the microsporidian from the Japanese anglerfish, they evidently belong to the same species. This report of Spraguea americana is the first report of a Spraguea species from L. litulon and indeed from the Pacific water mass.
\end{abstract}

Microsporidian parasites found in the nervous tissues of anglerfish of the genus Lophius were first reported by Thélohan (1895), who described the infection of the spinal ganglia of Lophius piscatorius (Linnaeus, 1758) collected from the Atlantic coast of France with a species of Glugea, but was unable to complete his description. Doflein (1898) found the same condition in $L$. piscatorius sampled from Italy, and as the parasite assemblages resembled those described by Moniez (1887) for Glugea anomala, infecting the musculature of the stickleback Gasterosteus aculeatus, the microsporidian from $L$. piscatorius was assigned to this genus and named Glugea lophii Doflein, 1898. Doflein mistakenly interpreted the host-parasite relationship and believed that infected host cells disintegrated to form large colonies of parasites; he did, however, correctly observe spore size and shape differences and cyst zonation, which he suggested was due to the differential pressures experienced by spores from various locations within the cyst. More detailed studies on G. lophii were conducted by Mrázek (1899), who described the hypertrophy of infected ganglion cells and the formation of cysts in the extended section (axon) of the cell, and he also described two zones in the cysts, which he called the darker outer zone and light inner zone and suggested that this was due to a fundamental difference in the material properties of each zone.

Pace (1908) and Weissenberg (1911a) independently changed the name of the parasite to Nosema lophii, the latter author believing a transfer to the genus Nosema was warranted due to the lack of a sporont stage during the developmental cycle, which conformed to the description of the genus at the time.

Weissenberg (1909, 1911a, b, c, 1976) essentially agreed with Mrázek's earlier work but he described xenoma formation in the cell body at the point where the axon is formed and not in the axon itself; he referred to this as the distal part of the cell body with the uninfected proximal zone containing the host cell nucleus. This type of host cell-parasite relationship is described by Weissenberg (1976) as unique, stressing that 1) the formation of parasite colonies are in the cytoplasmic zone of the host cell where the infection originated and 2) that meronts in the process of multiplication are found in the median layer of the cysts. Sprague and Vávra in a footnote to Weissenberg's paper (1976) mentioned for the first time the name "Spraguea g. n." with S. lophii as type species to replace Nosema lophii, thus becoming the authors of the new genus Spraguea Sprague et Vávra, 1976. Weissenberg in the series of papers (1909-1911c) also commented upon two regions of the cyst containing different spore forms; the external zone with oval spores and the internal zone with cylindrical spores, and suggested that the oval spores transform into cylindrical spores as the cysts mature as he observed fewer cylindrical spores in young cysts whilst mature cysts appeared to be dominated by them.

The first detailed ultrastructure study was carried out by Loubès et al. (1979); they studied Spraguea lophii from the nervous systems of both species of European

This paper was presented at the 6th International Symposium on Fish Parasites in Bloemfontein, South Africa, 22-26 September 2003. 
anglerfish, Lophius budegassa (Spinola, 1807) and $L$. piscatorius caught from the Atlantic and Mediterranean coasts of France. Loubès et al. (1979) described the xenomas as always containing two well-defined areas, each with a different developmental sequence and spore form. The outer peripheral area resulting in the production of oval spores containing a single nucleus 'type Nosemoides' spores, whilst the inner area resulted in the production of cylindrical spores with a diplokaryotic nuclear arrangement, 'type Nosema' spores. Takvorian and Cali (1986) conducted ultrastructure studies on neural microsporidian xenomas from the American anglerfish, Lophius americanus (Valenciennes, 1837), caught from the northeast Atlantic coast of the USA, but found only one spore form with a single nucleus. Due to the lack of spore dimorphism they reverted to the original generic classification of Doflein's and named the parasite from the American anglerfish, Glugea americanus (Takvorian and Cali 1986). Jakowska (1966) reported the presence of a similar microsporidian parasite in the nervous tissues of the South American anglerfish, Lophius gastrophysus (Miranda-Ribeiro, 1915), caught off the Brazilian and Venezuelan costs and described a similar histopathology, but lack of ultrastructure data prevented detailed comparisons to be made.

\section{MATERIALS AND METHODS}

A total of 10 Lophius litulon measuring from 27 to $54 \mathrm{~cm}$ in total length were sampled from fish markets and trawls. The fish were collected from four geographically distinct locations around the coast of Japan, including samples from both the Sea of Japan and the Pacific Ocean. Samples were taken between December 2002 and April 2003.

Fresh spore preparations were examined with a compound microscope and dimensions taken with an ocular micrometer. Dissected xenomas were fixed in $10 \%$ buffered formalin for 24 hours, dehydrated through a graded ethanol series, embedded in paraffin wax and sectioned at $5 \mu \mathrm{m}$. The sections were stained with haematoxylin and eosin (H\&E) and Uvitex 2B (Yokoyama et al. 1996). Sections were examined for the presence of spores with a fluorescence microscope utilising a 100 W mercury bulb (ultra violet excitation: $330-380 \mathrm{~nm}$ ).

Xenomas were fixed in $2.5 \%$ glutaraldehyde in $0.1 \mathrm{M}$ sodium cacodylate buffer ( $\mathrm{pH} 7.2)$ at $4^{\circ} \mathrm{C}$ for 2 hours. Specimens were then rinsed in $0.1 \mathrm{M}$ sodium cacodylate buffer at $4^{\circ} \mathrm{C}$ overnight before being post-fixed in $1 \%$ osmium tetroxide for one hour, dehydrated through an ethanol series, embedded in Spurr resin and polymerized at $60^{\circ} \mathrm{C}$ for 48 hours. Semithin sections of $1 \mu \mathrm{m}$ were first cut and visualised by staining with $0.5 \%$ toluidine blue. Ultrathin sections $(60-80 \mathrm{~nm})$ were mounted on 200 mesh, Formvar (Nisshin EM, Japan) coated copper grids and double stained with uranyl acetate and lead citrate. Ultrathin sections were examined using an electron microscope (JEM 1010, JEOL) at $80 \mathrm{kV}$.

Xenomas were homogenized in $0.5 \mathrm{ml}$ high concentration urea buffer (TNES-Urea: $10 \mathrm{mM}$ Tris- $\mathrm{HCl} \mathrm{pH} 7.5,125 \mathrm{mM}$ $\mathrm{NaCl}, 10 \mathrm{mM}$ EDTA, 0.5\% SDS, $4 \mathrm{M}$ urea; modified from Asahida et al. 1996), and vortexed (in a manner to maximise shearing directions) for $1 \mathrm{~min}$ with $0.4 \mathrm{~g}$ of $0.5 \mathrm{~mm}$ silica beads to facilitate the mechanical disruption of mature spore walls. Proteinase K was added to a concentration of $100 \mu \mathrm{g} / \mathrm{ml}$ and digestion allowed to occur overnight at $56^{\circ} \mathrm{C}$. DNA was subsequently extracted using a QIAamp DNA Mini Kit (Qiagen) following the manufacturer's tissue protocol; the purified DNA was used as template DNA for subsequent PCR reactions.

Targeted small subunit (SSU) rDNA was amplified using the PCR primers previously described by Pomport-Castillon et al. (1997); forward primer (5'-GGTTGATTCTGCCTGA CGT-3'), reverse primer (5'-GACGGGCGGTGTGTACA AAG-3').

Each $20 \mu \mathrm{l}$ PCR reaction contained $\sim 10 \mathrm{ng}$ of genomic DNA, $15 \mathrm{pmol}$ of each primer, $0.25 \mathrm{mM}$ of each dNTP, PCR buffer with a final magnesium concentration of $2 \mathrm{mM}$ and 0.5 units of Taq DNA polymerase (Takara Shuzo, Japan). After an initial denaturation at $95^{\circ} \mathrm{C}$ for $4 \mathrm{~min}$, samples were subjected to 30 cycles of amplification (denaturation at $95^{\circ} \mathrm{C}$ for $30 \mathrm{sec}$, primer annealing at $55^{\circ} \mathrm{C}$ for $30 \mathrm{sec}$, and extension at $72^{\circ} \mathrm{C}$ for $1 \mathrm{~min}$ ), followed by a $7 \mathrm{~min}$ terminal extension at $72^{\circ} \mathrm{C}$. All amplifications were performed on a Perkin-Elmer GeneAmp PCR System 2400 thermocycler. The PCR products obtained were visualised in an ethidium bromide-stained $1 \%$ agarose gel. PCR amplicons were purified using a PCR purification kit (Qiagen) and the resulting purified DNA used as template DNA in direct sequencing reactions or cloned into a vector. PCR amplicons were cloned into pt-7 Blue T-vector system (Takara Shuzo, Japan). Recombinant plasmids and PCR products were sequenced by the dideoxy chain termination method (Sanger et al. 1977) using a BigDye ${ }^{\mathrm{TM}}$ Terminator v3.1 Cycle Sequencing Ready Reaction Kit, and a 310 capillary DNA sequencer (Applied Biosystems), according to the manufacturer's instructions. To enable accurate sequencing of the complete SSU further oligonucleotide primers were designed: internal forward (350-fwd) (5'-ATTACCGAAGCC TCCAAGAGG-3'), internal reverse (870-rev) (5'-GTTGAG TCAAATTAAGCCGCA-3') which utilising the same PCR conditions amplify an internal amplicon of approximately 540 bases. DNA sequencing was performed on four fish, one from each location. Sense and anti-sense strands were sequenced for all products and four replicates (PCR products amplified from separate DNA extractions) performed for each gene region. The consensus sequence obtained was compared to other sequences available in the GenBank databases using BLAST (Altschul et al. 1990). CLUSTAL X (Thompson et al. 1997) was used for initial sequence alignments with default settings for gap and weight values. Alignment files were converted into distances by the Kimura- 2 parameter and trees constructed using the neighbour-joining (N-J) algorithm (Saitou and Nei 1987) in the TreeView 1.6.6 tree building software package (Page 1996).

The sequence determined in the current study was submitted to GenBank and assigned the accession number: AJ465876. GenBank accession numbers of additional sequences utilised in the analyses are: Glugea anomala AF056016, Glugea atherinae U15987, Glugea stephani AF056015, Heterosporis sp. AF356225, Ichthyosporidium sp. L39110, Kabatana takedai AF356222, Loma acerinae AJ252951, Microgemma caulleryi AY033054, Nosema apis 
U26534, Nucleospora sp. AF186007, Nucleospora salmonis AF185987, Ovipleistophora mirandellae AF356223, Pleistophora anguillarum U47052, Pleistophora mirandellae AJ252954, Pleistophora ovariae AJ252955, Pseudoloma neurophilia AF322654, Spraguea spp. AF104086, AF056013, AF056014 and AF033197, and Tetramicra brevifilum AF364303.

\section{RESULTS}

From the ten Lophius litulon sampled, all were visibly infected by a microsporidian parasite of the nervous tissue. Fresh squash preparations of dissected cysts revealed many elongate oval spores, some slightly curved, but of uniform size (Fig. 1). The average dimension of 50 spores was $3.4 \times 1.8 \mu \mathrm{m}$, larger macrospores were occasionally observed with an average dimension of 4.3 $\times 2.0 \mu \mathrm{m}$.

Infection was predominately observed in the vagal nerves near the kidneys (Fig. 2), but was also seen in the trigeminal nerves and spinal nerves of the vertebral column (Fig. 3); infection was also observed on brain tissue, being restricted to the dorsal surface of the medulla oblongata region of the hind brain. Assemblages of xenomas ranged in size from 1 to $20 \mathrm{~mm}$ in diameter, each containing many smaller xenomas, in the 'bunch of grapes-like' appearance first described by Doflein (1898). Infection was often observed to be asymmetrical, with heavy infections often occurring on one side of the body and only a light or no infection at all in the complementary region of the opposite side of the body; this was most often seen with infections of the vagal nerves near the kidney (Fig. 4) but also occasionally occurred in the spinal nerves along the length of the vertebral column. Fig. 5 depicts the ventral skeletal region where the spinal column fuses with the cranial plate; nerves emerging through the cranial plate and exiting from the posterior region of the plate are greatly enlarged with microsporidian masses.

Histological sections of xenomas revealed a significant hypertrophy in infected host ganglion cells both in the cytoplasmic and nuclear regions with the distal portion of the host cell containing the xenoma. Figs. 6 and 7 are the same section viewed under normal and UV light respectively and clearly demonstrate the xenomas, which are restricted to the distal part of the host cell. Viewed under fluorescent light, the chitin in the mature spore wall stains a bright blue-white, areas of nonstaining in both Figs. 7 and 8 represent developing and divisional stages of the parasite that have not yet formed a spore wall, typically seen in the median layer of the xenomas. Transmission electron microscopy of these non-staining areas revealed many multinucleate divisional plasmodia and developing sporoblasts (Fig. 9) which were found to be in direct contact with the host cell cytoplasm. Neither developing stages nor mature spores were observed enclosed in either a host- or parasite-derived interfacial envelope. Fig. 10 depicts a

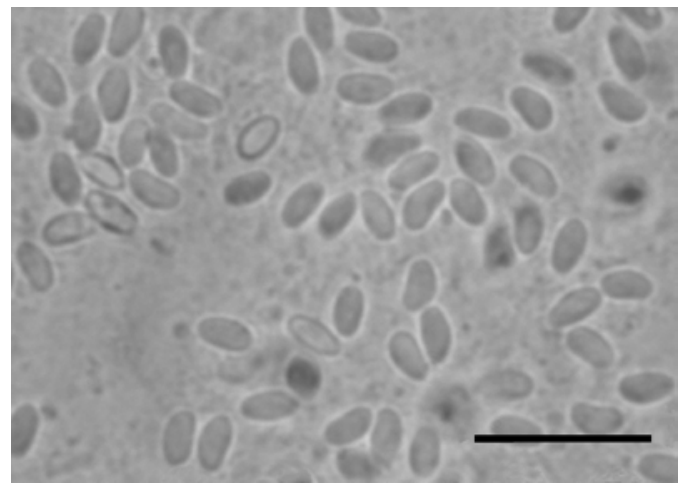

Fig. 1. Light micrograph of fresh microsporidian spores from infected nervous tissue of Lophius litulon. Scale bar $=10 \mu \mathrm{m}$.

ribbon-like plasmodium with four nuclei visible; the plasmodium contains filamentous endoplasmic reticulum (ER) and numerous ribosomes dispersed freely in the cytoplasm. Fig. 11 shows part of a plasmodium undergoing division; the cytoplasm appears heavily vacuolised with ER during this divisional stage. The onset of sporogony is characterised by the thickening of the plasmodial membrane through the addition of dense vesicles to the outer surface; the ER is now in wide cisternae containing many bound ribosomes (Fig. 12). After progressive fragmentation of the plasmodia into smaller multinucleate stages and final fission into cells containing a single nucleus, immature sporoblasts are formed (Fig. 13), after which no further cell division occurs. At this stage the cell membrane thickens further staining very electron-dense; large electron-lucent vesicles (500 nm in diameter) are present in the immature sporoblasts which are precursors to the spore organelles. As the sporoblasts develops further (Fig. 14), the polar tube, a large posterior vacuole and the lamellar polaroplast become apparent. The nucleus is centrally positioned between the posterior vacuole and the polaroplast, which is divided into two zones. The polar tube cross-sections reveal an electron-dense core, the electron-lucent endospore is forming and a degree of surface ornamentation is already present. Mature spores (Figs. 15-18) have an electron-dense exospore wall that is 25$35 \mathrm{~nm}$ thick and has a pyramidal-shaped ornamentation on the outer surface. The ornamentation, although not always clearly visible, was observed to be present uniformly over the spores' surface; the distance between the apexes of the pyramidal ridges is approximately 40 $\mathrm{nm}$ (Fig. 18). The electron-lucent endospore wall is 50 $80 \mathrm{~nm}$ thick, narrowing significantly to $20 \mathrm{~nm}$ at the anterior of the spore at the attachment site of the polar tube, which is often radically apically shifted (Figs. 15, 16). The polaroplast is of two distinct types; the anterior type is of a fine lamellar structure with approximately 10 $\mathrm{nm}$ between the lamellar folds (Fig. 15); this fine lamellar polaroplast traverses the entire anterior portion of the spore occupying about $20-25 \%$ of the total spore 


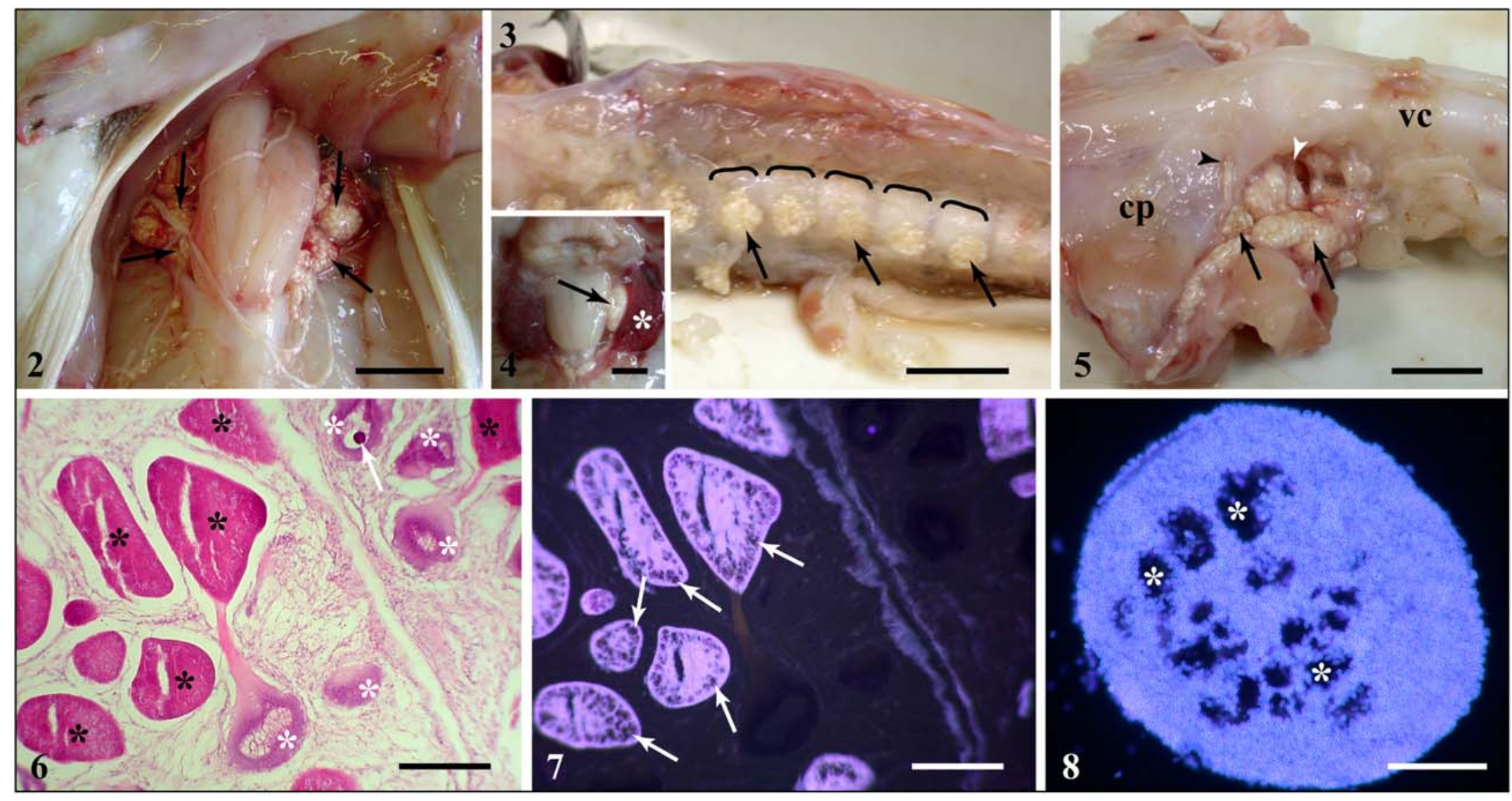

Figs. 2-8. The cellular location of the microsporidian in the nervous tissue of Lophius litulon. Fig. 2. A ventral dissection of the fish reveals large assemblages of xenomas in the vagal nerves (black arrows) situated near the kidneys (removed). Fig. 3. Groups of xenomas (black arrows) developing in spinal nerves emerging from individual vertebra (five marked with brackets). Fig. 4. (Inset Fig. 3) Fish showing a xenoma formation (black arrow) near the left kidney (white asterisk), while no infection exists in the right-side vagal nerve complex. Fig. 5. Skeletal ventral surface where the vertebral column (vc) fuses with the cranial plate (cp), (tissues removed) showing the vagal (white arrowhead) and glossopharyngeal (black arrowhead) nerves exiting the central nervous system, greatly enlarged with microsporidian infection (black arrows). Figs. 6, 7. Histological section stained with H\&E and Uvitex 2B. Fig. 6. Viewed using conventional light microscopy; black asterisks indicate xenomas which are restricted to the distal zones of hypertrophic host ganglion cells. The uninfected part of the cell indicated by the white asterisks contains the nucleus (white arrow); both the uninfected area of the host cell and the host nucleus are also significantly hypertrophic. Fig. 7. Same section viewed in UV light only; shows the microsporidian spores staining a bright blue-white colour, the xenomas being restricted to the distal zone of the host cell; white arrows represent non-staining pre-spore stages. Fig. 8. Mature xenoma stained with Uvitex 2B; non-staining areas (white asterisk) of the xenoma represent developing stages of the parasite, also visible in Fig. 7 (white arrows). Scale bars: Figs. 2, 3, $5=20$ mm; Fig. $4=10 \mathrm{~mm}$; Figs. $6,7=200 \mu \mathrm{m}$; Fig. $8=50 \mu \mathrm{m}$. 

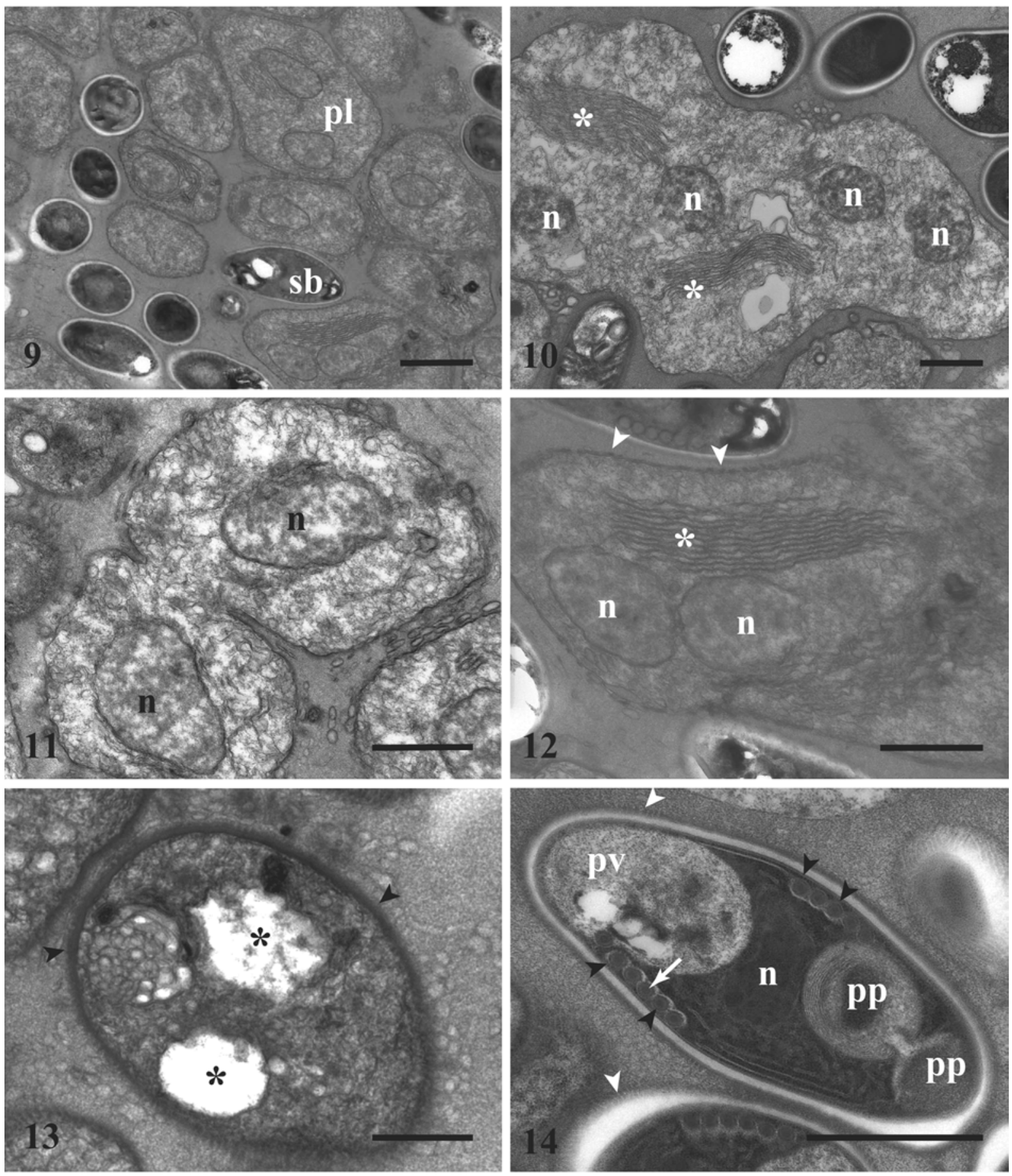

Figs. 9-14. Transmission electron micrographs of the microsporidian developing pre-spore stages in the nervous tissue of Lophius litulon. Fig. 9. Plasmodia (pl) and sporoblasts (sb) develop in direct contact with the host cell cytoplasm. Fig. 10. A plasmodium with four nuclei (n) and fine filamentous endoplasmic reticulum (ER) (white asterisks). Fig. 11. A divisional plasmodium with two nuclei (n) visible; the cytoplasm is heavily vacuolised during division. Fig. 12. A transforming plasmodium with two nuclei (n) visible; the ER (white asterisk) is now in the form of much wider cisternae; a thick dense cell coat is forming by the acquisition of dense vesicles on the cell membrane (white arrowheads). Fig. 13. An early sporoblast formed after the recent multiple fission of a sporogonial plasmodium; a thick spore wall is forming (black arrowheads), the electron-lucent vesicles (black asterisks) are precursors to the spore organelles. Fig. 14. A late sporoblast; the developing polaroplast (pp) is in two distinct zones; a single central nucleus (n) and posterior vacuole (pv) can be easily distinguished. Six turns of the polar filament are visible (black arrowheads), the cross-section of which details an electron-dense core (white arrow), spore surface ornamentation is already apparent (white arrowheads). Scale bars: Fig. $9=2 \mu \mathrm{m}$; Figs. $10-12,14=1 \mu \mathrm{m}$; Fig. $13=500 \mathrm{~nm}$. 

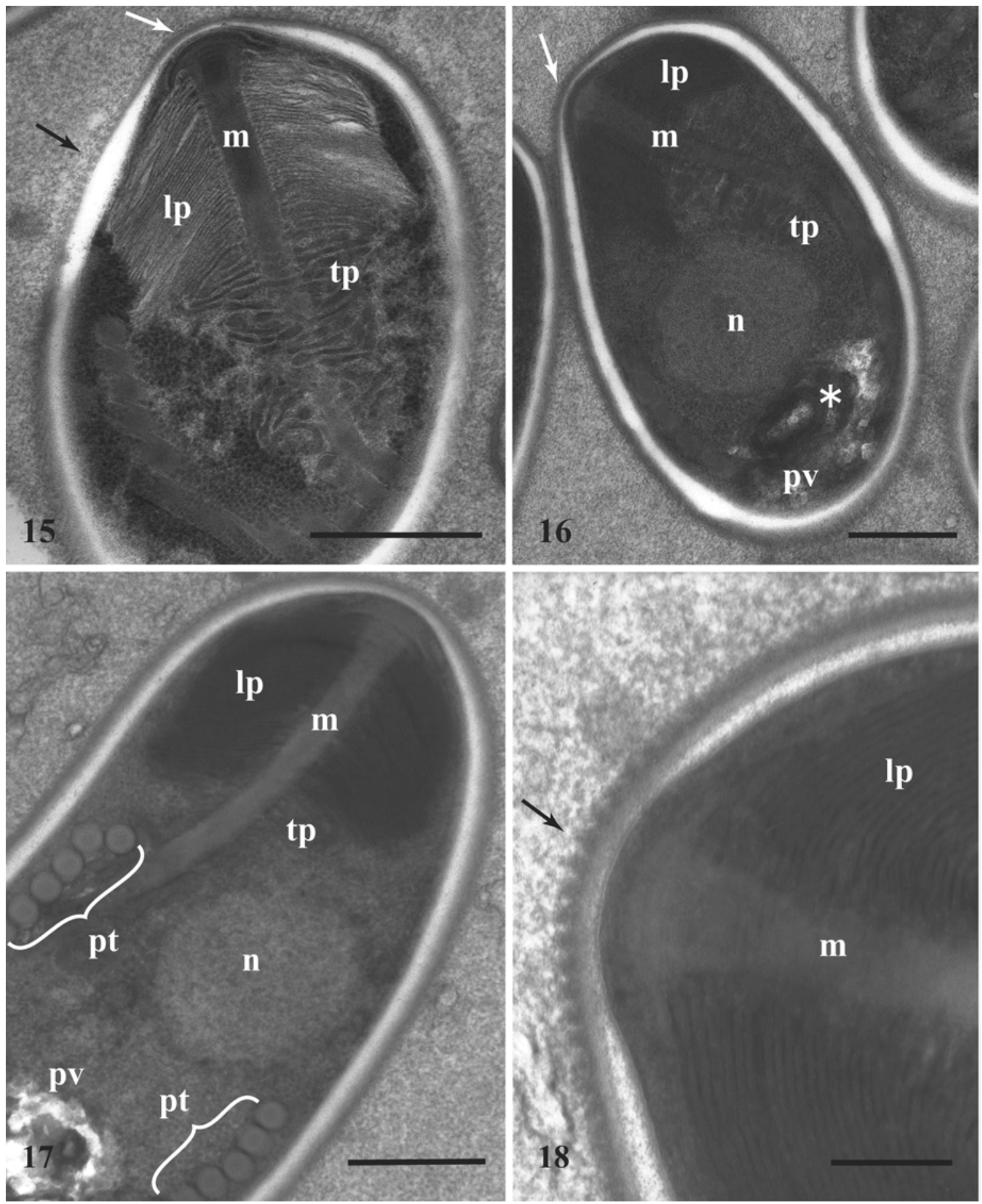

Figs. 15-18. Transmission electron micrographs of mature spores of the microsporidian from Lophius litulon. Spores show two clear arrangements of polaroplast, an anterior laminar type (lp), and posterior tubular type (tp) which is closely associated with the manubroid part of the polar tube (m). Note the conspicuous narrowing of the endospore (white arrows) at the anchoring disc of the polar tube. The single nucleus (n) centrally positioned and a large membranous (white asterisk) body (Fig. 16) is situated in the polar vacuole (pv). The manubroid part of the polar tube extends past the last anterior coil of the polar tube (pt), suggesting a coiling direction from the posterior end of the spore towards the anterior. Spore surface ornamentations are present in the form of pyramidal ridges (black arrows). Scale bars: Figs. $15-17=500 \mathrm{~nm}$; Fig. $18=200 \mathrm{~nm}$. 


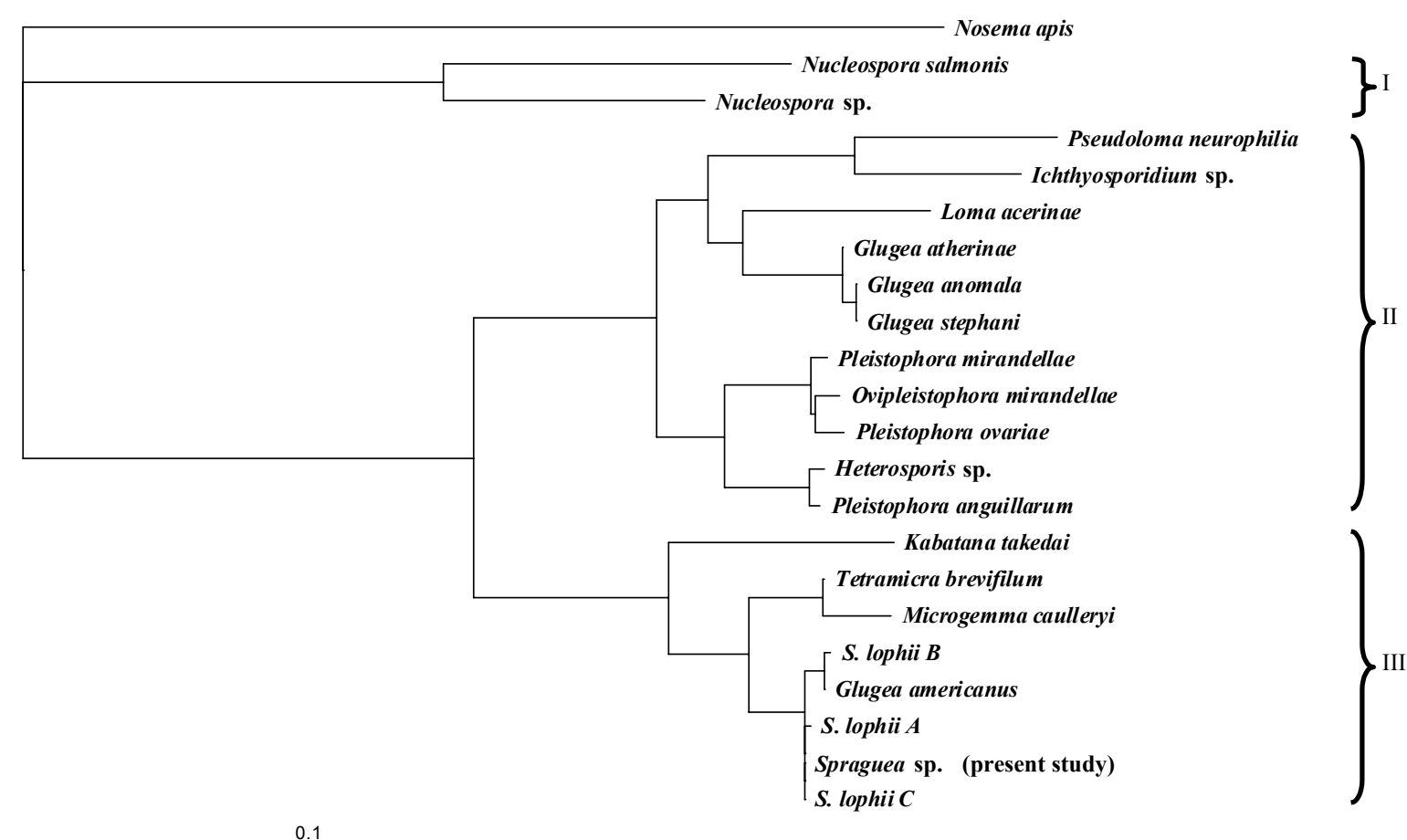

Fig. 19. Neighbour-joining phylogram of 21 fish-infecting microsporidia. The tree is rooted to a distantly related outgroup, Nosema apis, infecting an insect. The tree forms three main clades; clade I includes members of the genus Nucleospora; clade II represents other fish-infecting microsporidia, including the genera Loma, Glugea and Pleistophora; clade III includes the sequence from the present study and all other isolates of Spraguea spp., plus representatives of the genera Tetramicra, Microgemma and Kabatana. Genetic distance is represented by the scale bar.

volume. The more posteriorly positioned polaroplast is very coarse in comparison and with a far less organised arrangement; the folds appear tubular in shape with a diameter of approximately $20-25 \mathrm{~nm}$. This tubular-type polaroplast does not traverse the entire width of the spore and is more closely associated with the polar tube and may persist along the remaining manubroid portion of the tube (Fig. 15). The polar tube is isofilar with between 5 and 8 (predominately 6) coils arranged in a single layer. The manubroid portion of the polar tube may extend almost the entire length of the spore and is approximately $115 \mathrm{~nm}$ thick (Fig. 15), the cross-section of the coiled part of the polar tube is between 100-115 $\mathrm{nm}$ in diameter and stains more electron-dense in the centre. The single nucleus is centrally positioned within the spore and ranges between 600 and $750 \mathrm{~nm}$ in diameter and stains less electron-dense than the ribosomerich spore cytoplasm. The posterior vacuole is positioned at the posterior end of the spore and stains very electron-lucent in areas; it also contains a large membranous structure between 200 and $400 \mathrm{~nm}$ in size. A comparison of the spores' dimensions and characteristic features from this study compared to those from both the American and European anglerfish are given in Table 1.

Phylogenetic analysis of the small subunit (SSU) rDNA obtained from the microsporidian from Japanese anglerfish and 20 other fish-infecting microsporidia re- vealed an extremely close relationship to the SSU sequences from both European and American anglerfish species (listed in Table 2), with $>99.5 \%$ identities to all sequences. These small differences are represented by $<10$ gap or base insertions; or between 1 and 3 base transitions or transversions (Table 3) present along the entire SSU sequences analysed. Fig. 19 details the phylogenetic relationship between the other microsporidians used in the analysis and the genus Spraguea. The phylogram forms three distinct clades, which are all supported by $100 \%$ nodal bootstrap values (data not shown). Clade I represents the most distantly related microsporidia of the genus Nucleospora that infect fish which have $<70 \%$ sequence identities to the members of the genus Spraguea. Clade II represents other fishinfecting microsporidia including the less distantly related species, Glugea anomala, Pleistophora mirandellae and Ichthyosporidium sp., which have distance values/percentage identities to the sequence obtained in this study of $0.1285 / 87.2 \%, 0.1273 / 87.3 \%$ and $0.1701 /$ $83 \%$, respectively. Clade III includes all the known sequences from the microsporidians infecting the nervous system of lophiid fish, plus members of the genera Kabatana, Tetramicra and Microgemma, which have distance values/percentage identities to the sequence obtained in this study of $0.0694 / 93.1 \%, 0.0278 / 97.2 \%$ and $0.0544 / 94.6 \%$, respectively. 


\section{DISCUSSION}

Analyses of the microsporidian spores from the nervous tissues of Lophius litulon reveal many similarities to the uninuclear forms of Spraguea spp. seen in both European and American lophiid fish. Unfixed spore dimensions are almost identical to the European uninuclear spores and are only slightly larger than the American spores (Table 1). However, all uninuclear forms have a similar spore surface ornamentation and a comparable number of turns to the polar tube. The diplokaryotic spore form reported only in European lophiids forms the exception, which has no surface ornamentation, different dimensions and fewer turns of the polar tube. Other ultrastructural similarities also exist between the uninuclear forms; Loubès et al. (1979) described from European lophiids a similar polysporous developmental sequence with a multinucleate sporogonial plasmodium giving rise to several sporoblasts. Pre-spore stages were not described by Takvorian and Cali (1986) from the American anglerfish; however, their detailed description of the mature spore included many features also seen in the present study, including: polaroplast consisting of a lamellar anterior type and a tubular posterior type, a long manubroid portion of the polar tube which may be seen to traverse the entire length of the spore, and a large membranous structure contained within the posterior vacuole.

The location of the microsporidian infection in the Japanese anglerfish is extremely similar to that described from both European and American lophiids (Weissenberg 1911c, 1976, Jakowska 1964, Takvorian and Cali 1986); infections being found in the trigeminal nerves, spinal nerves of the vertebral column, vagal nerves near the kidney and on the medulla oblongata region of the hind brain. However, infection was never observed on the optic chiasma as described from the American anglerfish (Takvorian and Cali 1986). Histological examination of infected tissues also revealed very similar infections in the nerve ganglia to those reported from other lophiid species, with the xenomas being restricted to the distal portion of vastly hypertrophied host nerve cells (Mrázek 1899, Weissenberg 1911c, 1976, Jakowska 1964). However, the distinct spore zonation patterns present in the xenomas from European anglerfish were never observed in the Japanese species and any heterogeneous nature to the xenomas was attributed to the developing stages of the uninucleate spores, often observed at the median layer in the xenoma, as described by Weissenberg (1976).

Molecular phylogeny based on SSU rDNA sequences demonstrates an extremely close relationship amongst all the known gene sequences from Spraguea infections in lophiid fish from Europe, Japan and America, with all sequences having $>99.5 \%$ identities. In some cases the differences are only due to a few sequence deletions/ insertions which could easily be explained by sequencing or amplifications errors. This result may seem sur- prising due to the geographical isolation of the fish species and the ultrastructural differences seen in the European species. The sequences from Spraguea spp. form a monophyletic clade with members of the family Tetramicridae and a sister group to that containing $\mathrm{Ka}$ batana takedai. This phylogenetic grouping was consistently achieved and is in agreement with Lom and Nilsen's (2003) review, which also grouped these genera together. Although members of the Tetramicridae are xenoma-forming microsporidia that infect marine fish, they have never been reported from nervous tissue and are predominately found infecting hepatocytes and skeletal muscles. Some ultrastructural comparisons can be made between the microsporidian spores from the Japanese anglerfish and members of the Tetramicridae. Tetramicra brevifilum has uninuclear spores, only slightly larger in size, with two distinct layers of polaroplast and a large inclusion body in the posterior vacuole, and has also been reported infecting the musculature of Lophius budegassa (Matthews and Matthews 1980, Maíllo et al. 1998). Microgemma ovoidea is polysporous, has a similar spore size, with a comparable number of turns to the polar tube and also has polaroplast arranged in two distinct zones (Amigó et al. 1996).

Loubès et al. (1979) suggested two explanations for the spore dimorphism in European lophiids; that the two spore forms may represent two separate species of microsporidian or that the dimorphism was due to a single species of microsporidians entering a sexual phase of reproduction or responding in some way to external environmental conditions. They also commented upon the fact that the dimorphic condition was always present but to varying degrees within given xenomas, a condition also observed by Weissenberg (1976) and considered by him to be due to xenoma maturation. Assumptions can be made that during the SSU sequencing of Spraguea lophii from European lophiids by Nilsen (1999) and Pomport-Castillon et al. (2000), the presence of two species of microsporidians would have become apparent, therefore the dimorphic infection in European lophiids must be considered to be caused by a single species of microsporidian. As this dimorphic condition is never present in the Japanese and American anglerfish species, but is a consistent feature in the European species, the microsporidia from both the Japanese and American anglerfish species should be currently considered as a separate species from $S$. lophii infecting European anglerfish despite having an almost identical SSU rRNA gene sequence. Assuming the microsporidian parasites in European, American and Japanese Lophius spp. represent discrete populations and are not subject to global distribution by an unknown ubiquitous alternate or intermediate host, the evolutionary rate of change with respect to the SSU rRNA gene is unexpectedly low and other less conserved genes should be analysed which may give some indication of evolutionary variation within the genus. 
Table 1. Spore dimensions and characteristic features of Spraguea spp. from European, American and Japanese Lophius spp. Note that two spore forms are represented from both species of European anglerfish.

\begin{tabular}{|l|c|c|c|c|}
\hline & Lophius americanus & \multicolumn{2}{c|}{$\begin{array}{c}\text { Lophius piscatorius } \text { and } \\
\text { Lophius budegassa }\end{array}$} & Lophius litulon \\
\hline Spore dimorphism & absent & \multicolumn{2}{|c|}{ present } & absent \\
\hline Nuclear arrangement & uninuclear & $\begin{array}{c}\text { uninuclear } \\
\text { 'Nosemoides' }\end{array}$ & $\begin{array}{c}\text { diplokaryotic } \\
\text { 'Nosema' }\end{array}$ & uninuclear \\
\hline $\begin{array}{c}\text { Average spore } \\
\text { dimensions }\end{array}$ & $2.8 \times 1.5 \mu \mathrm{m}$ & $3.5 \times 1.5 \mu \mathrm{m}$ & $4 \times 1.25 \mu \mathrm{m}$ & $3.4 \times 1.8 \mu \mathrm{m}$ \\
\hline $\begin{array}{c}\text { Number of coils of } \\
\text { polar tube }\end{array}$ & $6-9$ & $5-6$ & $3-4$ & $5-8$ \\
\hline $\begin{array}{c}\text { Spore surface } \\
\text { ornamentation }\end{array}$ & present & present & absent & present \\
\hline Reference & Takvorian and Cali 1986 & Loubès et al. 1979 & Loubès et al. 1979 & present study \\
\hline
\end{tabular}

Table 2. A list of the small subunit rDNA sequences from different isolates of Spraguea spp., taken from different Lophius species, used in the phylogenetic analysis.

\begin{tabular}{|l|c|c|c|}
\hline $\begin{array}{c}\text { Sequence identity and } \\
\text { GenBank accession no. }\end{array}$ & Host fish & $\begin{array}{c}\text { Length of SSU } \\
\text { used in analysis }\end{array}$ & Reference \\
\hline $\begin{array}{l}\text { Spraguea lophii A } \\
\text { AF104086 }\end{array}$ & L. piscatorius & 1239 & Nilsen 1999 \\
\hline $\begin{array}{c}\text { Spraguea lophii B } \\
\text { AF056013 }\end{array}$ & $\begin{array}{c}\text { L. piscatorius } \\
\text { L. budegassa }\end{array}$ & 1065 & $\begin{array}{c}\text { Pomport-Castillon et al. } \\
2000\end{array}$ \\
\hline $\begin{array}{c}\text { Spraguea lophii C } \\
\text { AF033197 }\end{array}$ & L. americanus & 1252 & Hinkle et al. 1997 \\
\hline $\begin{array}{c}\text { Glugea americanus } \\
\text { AF056014 }\end{array}$ & L. americanus & 1064 & $\begin{array}{c}\text { Pomport-Castillon et al. } \\
2000\end{array}$ \\
\hline $\begin{array}{c}\text { Spraguea } \text { sp. } \\
\text { AY465876 }\end{array}$ & L. litulon & 1244 & present study \\
\hline
\end{tabular}

Table 3. Pairwise distance data and relative percentage identities obtained by Kimura-2 parameter analysis of the different isolates of Spraguea spp., with respect to the sequence obtained during this study of the microsporidian from the Japanese anglerfish. Included are the number of gaps, insertions, transitions and transversions that account for these sequence differences.

\begin{tabular}{|l|c|c|c|c|c|}
\hline \multicolumn{1}{|c|}{ Sequence } & $\begin{array}{c}\text { Pairwise distance data and } \\
\text { percentage identity }\end{array}$ & Gaps & Insertions & Transitions & Transversions \\
\hline S. lophii A & $0.0023 \equiv 99.77 \%$ & 7 & 2 & 0 & 0 \\
S. lophii B & $0.0035 \equiv 99.65 \%$ & 0 & 0 & 3 & 2 \\
S. lophii C & $0.0000 \equiv 100 \%$ & 0 & 8 & 0 & 0 \\
G. americanus & $0.0035 \equiv 99.65 \%$ & 2 & 6 & 1 & 2 \\
\hline
\end{tabular}

The authors agree with the earlier findings of Pomport-Castillon et al. (2000), Nilsen (2000) and Lom and Nilsen (2003) that the parasite from the American anglerfish, described as Glugea americanus (Takvorian and Cali 1986), should be transferred to the genus Spraguea as Spraguea americana comb. n. and the description of the genus Spraguea be amended to include such monomorphic forms. At the present time no major differences in ultrastructure or known gene sequence data exist between Spraguea americana from Lophius americanus and the microsporidian from the Japanese anglerfish and hence they should be considered the same species, $S$. americana.

The prevalence of $S$. americana from the Japanese anglerfish sampled during this study was extremely high $(100 \%)$, however the degree of infection was very variable, with some fish only containing very few small xenomas whilst others had numerous large assemblages of xenomas. The location and degree of infection in the fish did not appear to be linked to fish size or the loca- tion of capture. No serious clinical signs from infection were evident and the fish were not considered to be emaciated, however no uninfected fish were sampled for direct comparisons to be made. The prevalence of Spraguea infections reported from previous studies in Europe and the USA have been recorded as high as $100 \%$ in Lophius piscatorius caught from the Atlantic coast of France and L. americanus taken from the New York Bight area (Loubès et al. 1979, Jakowska 1966), but Loubès et al. (1979) suggested that the prevalence of the parasite was in decline in the Mediterranean Sea and American lophiid populations, compared to that from earlier studies. No convincing scientific data exist to suggest that Spraguea spp. produce any disease condition in lophiid fishes, which seems surprising due to the extensive infections observed, causing extreme hypertrophy to nerves involved in innervating vital organs. Further studies with more extensive sampling regimes could help to establish the detrimental effects $S$. americana has on its commercially important host, if any. 


\section{REFERENCES}

ALTSCHUL S.F., GISH W., MILLER W., MYERS E.W., LIPMAN D.J. 1990: Basic local alignment search tool. J. Mol. Biol. 215: 403-410.

AMIGÓ J.M., SALVADÓ H., GRACIA M.P., VIVARÉS C.P. 1996: Ultrastructure and development of Microsporidium ovoideum (Thélohan, 1895) Sprague, 1977, a microsporidian parasite of the red band fish (Cepola macrophthalma L.). Eur. J. Protistol. 32: 532-538.

ASAHIDA T., KOBAYASHI T., SAITOH K., NAKAYMA I. 1996: Tissue preservation and total DNA extraction from fish stored at ambient temperature using buffers containing high concentrations of urea. Fish. Sci. 62: 727-730.

DOFLEIN F. 1898: Studien zur Naturgeschichte der Protozoen. III. Über die Myxosporidien. Zool. Jahrb. Abt. Anat. 11: 281-350.

HINKLE G., MORRISON H., SOGIN M. 1997: Genes coding for reverse transcriptase, DNA-directed RNA polymerase, and chitin synthase from the microsporidian Spraguea lophii. Biol. Bull. (Woods Hole) 193: 250-251.

JAKOWSKA S. 1964: Infecção microsporídea das células nervosas numa população de peixes marinho, Lophius americanus. An. 2nd Congr. Lat.-Am. Zool. 1: 265-273.

JAKOWSKA S. 1966: Infection with neurotropic microsporidians in South American Lophius. Trans. Am. Microsc. Soc. 85: 161-162.

LOM J., NILSEN F. 2003: Fish microsporidia: fine structural diversity and phylogeny. Int. J. Parasitol. 33: 107-127.

LOUBÈS C., MAURAND J., ORMIÈRES R. 1979: Étude ultrastructurale de Spraguea lophii (Doflein, 1898), microsporidie parasite de la Baudroie: essai d'interprétation du dimorphisme sporal. Protistologica 15: 43-54.

MAÍLLO P.A., AMIGÓ J.M., BAENA R., SALVADÓ H., GRACIA M.P. 1998: Tetramicra brevifilum (Matthews \& Matthews, 1980) (Microsporida: Tetamicriidae) in a new fish host, Lophius budegassa (Spinola, 1807) in Spain. Parasitol. Res. 84: 208-212.

MATTHEWS R.A., MATTHEWS B.F. 1980: Cell and tissue reactions of turbot Scophthalmus maximus (L.) to Tetramicra brevifilum gen. n., sp. n. (Microspora). J. Fish Dis. 3: 495-515.

MONIEZ R. 1887: Observations pour la revision des Microsporidies. C. R. Acad. Sci. 104: 1312-1314.

MRÁZEK A. 1899: Sporozoenstudien II. Glugea lophii Doflein. Sitzungsber. Königl. Böhm. Ges. Wiss., Math.Naturwiss. Cl., Prag 34: 1-8.

NILSEN F. 1999: Small subunit rDNA phylogeny of Bacillidium sp. (Microspora: Mrazekiidae) infecting oligochaetes. Parasitology 118: 553-558.

NILSEN F. 2000: Small subunit ribosomal DNA phylogeny of microsporidia with particular reference to genera that infect fish. J. Parasitol. 86: 128-133.

PACE D. 1908: Parasiten und Pseudoparasiten der Nervenzelle. Vorläufige Mitteilungen über vergleichende Parasitologie des Nervensystems. Z. Hyg. 60: 62-74.
PAGE R.D.M. 1996: TREEVIEW: An application to display phylogenetic trees on personal computers. Comput. Appl. Biosci. 12: 357-358.

POMPORT-CASTILLON C., ROMESTAND B., De JONCKHEERE J.F. 1997: Identification and phylogenetic relationship of microsporidia by riboprinting. J. Eukaryot. Microbiol. 44: 540-544.

POMPORT-CASTILLON C., De JONCKHEERE J.F., ROMESTAND B. 2000: Ribosomal DNA sequences of Glugea anomala, G. stephani, G. americanus and Spraguea lophii (Microsporidia): phylogenetic reconstruction. Dis. Aquat. Org. 40: 125-129.

SAITOU N., NEI M. 1987: The neighbour joining method: a new method for reconstructing phylogenetic trees. Mol. Biol. Evol. 4: 406-425.

SANGER F., NICKLEN S., COULSON A.R. 1977: DNA sequencing with chain terminating inhibitors. Proc. Natl. Acad. Sci. USA 74: 5463-5467.

TAKVORIAN P., CALI A. 1986: The ultrastructure of spores (Protozoa: Microsporidia) from Lophius americanus, the anglerfish. J. Protozool. 33: 570-575.

THÉLOHAN P. 1895: Recherches sur les myxosporidies. Bull. Sci. Fr. Belg. 26: 100-394.

THOMPSON J.D., GIBSON T.J., PLEWNIAK F., JEANMOUGIN F., HIGGINS D.G. 1997: The CLUSTAL_X windows interface: flexible strategies for multiple sequence alignment aided by quality analysis tools. Nucleic Acids Res. 24: 4876-4882.

WEISSENBERG R. 1909: Beiträge zur Kenntnis von Glugea lophii Doflein. I. Über den Sitz und die Verbreitung der Mikrosporidien-Zysten am Nervensystem von Lophius piscatorius und L. budegassa. Sitzungsber. Ges. Naturforsch. Freunde Berlin 9: 557-567.

WEISSENBERG R. 1911a: Über einige Mikrosporidien aus Fischen (Nosema lophii Doflein, Glugea anomala Moniez, Glugea hertwigii nov. spec.). Sitzungsber. Ges. Naturforsch. Freunde Berlin. 8: 344-357.

WEISSENBERG R. 1911b: Beiträge zur Kenntnis von Glugea lophii Doflein. II. Über den Bau der Zysten und die Beziehungen zwischen Parasit und Wirtsgewebe. Sitzungsber. Ges. Naturforsch. Freunde Berlin. 3: 149-157.

WEISSENBERG R. 1911c: Über Mikrosporidien aus dem Nervensystem von Fischen (Glugea lophii Doflein) und die Hypertrophie der befallenen Ganglienzellen. Arch. Mikrosk. Anat. 78: 383-421.

WEISSENBERG R. 1976: Microsporidian interactions with the host cell. In: L.A. Bulla and T.C. Cheng (Eds.), Comparative Pathobiology. Volume I. Plenum Press, New York and London, pp. 203-237.

YOKOYAMA H., KIM J., SATO J., SANO M., HIRANO K. 1996: Fluorochrome Uvitex 2B stain for detection of the microsporidian causing beko disease of yellowtail and goldstriped amberjack juveniles. Fish Pathol. 31: 99-104. 